\title{
Impact of Nutrition Support Team on Postoperative Nutritional Status and Outcome of Patients with Congenital Gastrointestinal Anomalies
}

\author{
Parisa Zarei-shargh ${ }^{1}$, Emad Yuzbashian ${ }^{2}$, Atieh Mehdizadeh-Hakkak ${ }^{2}$, Zahra Khorasanchi ${ }^{2}$, \\ Abdolreza Norouzy ${ }^{3}$, Gholamreza Khademi ${ }^{4}$, Bahareh Imani ${ }^{4, *}$
}

1. Department of Nutrition, Varastegan Institute for Medical Sciences, Mashhad, Iran

2. Student Research Committee, Department of Nutrition, Mashhad University of Medical Sciences, Mashhad, Iran

3. Biochemistry and Nutrition Research Center and Department of Nutrition, Mashhad University of Medical Sciences, Mashad, Iran

4. Department of Pediatrics, Mashhad University of Medical Science, Mashhad, Iran

\section{* Corresponding Author:}

Bahareh Imani, MD Assistant Professor of Pediatrics, Pediatrics Department, Faculty of Medicine,

Mashhad University of Medical Science, Mashhad, Iran

Tel: + 989171118516

Fax: + 985138543031

Email: imanibh@mums.ac.ir

Received: 03 Nov. 2019

Accepted: 08 Feb. 2020

\section{ABSTRACT}

\section{BACKGROUND}

The aim of this study was to evaluate postoperative nutritional status in patients who underwent operations due to congenital gastrointestinal anomalies in surgical neonatal intensive care units (NICUs) and to investigate the role of nutrition support teams (NSTs) on the outcome.

\section{METHODS}

A retrospective clinical study was carried out at two NICUs in Dr. Sheikh Pediatric Hospital, Mashhad, Iran. One of the NICUs was supported by NST and the other was not. A total of 120 patients were included through a non-random simple sampling. Different variables such as age, sex, prematurity, type of anomaly, birth weight, use of vasoactive drugs, weight gain in NICU, length of NICU stay, postoperative enteral nutrition initiation, duration of mechanical ventilation, mortality rate, maximum of blood sugar, the amount of calorie delivered to the calorie requirement ratio, and distribution of energy from enteral or parenteral roots were compared between the patients of two NICUs.

\section{RESULTS}

Median weight gain and the amount of calorie delivered during NICU stay in subjects of NSTsupported NICU was significantly more than other NICU. There was no significant difference in the length of NICU stay, enteral nutrition initiation after the operation, ventilation days, and percent of mortality between the two groups. The percentage of enteral feeding was also increased by about $2.8 \%$, which was not significant.

\section{CONCLUSION}

NST could increase post-operative weight gain and calorie delivery in patients as well as providing an increase in enteral feeding rather than parenteral.

\section{KEYWORDS:}

Nutritional support, Digestive system abnormalities, Nutritional status, Neonatal intensive care units

\section{Please cite this paper as:}

Zarei-shargh P, Yuzbashian E, Mehdizadeh-Hakkak A, Khorasanchi Z, Norouzy AR, Khademi GR, Imani B. Impact of Nutrition Support Team on Postoperative Nutritional Status and Outcome of Patients with Congenital Gastrointestinal Anomalies. Middle East J Dig Dis 2020;12:116-122. doi: 10.34172/mejdd.2020.171.

\section{INTRODUCTION}

Congenital gastrointestinal (GI) malformations are developmental disorders or embryopathies, which may involve the gut or other systems. ${ }^{1}$ The prevalence of GI anomalies in Iran is very high (3.6 in 1000 live births). ${ }^{2}$ Surgical treatment is the usual intervention for the correction of GI anomalies and nutrition is the key component in postoperative management. The ability to sustain calorie 


\begin{tabular}{|c|c|c|}
\hline Daily Activities & Weekly Activities & Monthly Activities \\
\hline Nutritional assessment & Nutritional programs & Nutritional training for medical staff \\
\hline Clinical visit & Presentation of nutritional topics & \\
\hline $\begin{array}{l}\text { Administration, revision, and evaluation of enteral } \\
\text { and parenteral nutrition order }\end{array}$ & Nutritional training for dietitians & \\
\hline \multicolumn{3}{|l|}{ Monitoring of feeding tolerance } \\
\hline \multicolumn{3}{|l|}{ Control of fluid and electrolytes balance } \\
\hline \multicolumn{3}{|l|}{ Control of acid and base alteration } \\
\hline \multicolumn{3}{|l|}{ Control of metabolic factors and laboratory tests } \\
\hline \multicolumn{3}{|l|}{$\begin{array}{l}\text { Need assessment of energy and macronutrient } \\
\text { intake }\end{array}$} \\
\hline $\begin{array}{l}\text { Control of drug-drug interaction and food-drug } \\
\text { interaction }\end{array}$ & & \\
\hline
\end{tabular}

Fig.1: Activities of the nutrition support team in NICU of Dr. Sheikh Pediatric Hospital, Mashhad, Iran

intake in the setting of GI dysfunction is one of the important contributing factors in the improved outcome of neonates in the modern era. The provision of adequate calorie intake is under the influence of nutritive support. Moreover, minimal trace elements and micronutrient supplementation are included in early parenteral nutrition (PN) orders. ${ }^{3}$ Patients who undergo GI surgeries because of congenital anomalies need special nutritional care and management and should be closely supported by a nutrition support team (NST). ${ }^{4}$

Postsurgical nutritional support in neonates with congenital anomalies is different from adults and is complicated by prematurity, operative stress, critical illness, and sepsis. Moreover, adequate and even higher nutrition supply should be considered in these patients for postsurgical healing, and normal growth and development. ${ }^{5}$ Many studies demonstrate the cost-saving and improvement in patients' outcomes after the implementation of NST. ${ }^{6,7}$ American society for parenteral and enteral nutrition (Aspen) at last updated guideline reported the existence of NST in $42 \%$ of the respondent hospitals in $2010 .^{8}$

Nutrition support professionals include physicians, nurses, dietitians, and pharmacists who manage several issues regarding complete nutritional support such as appropriate nutritional assessment in each patient, assignment of macro- and micronutrient requirement, administration of proper enteral and parenteral nutrition, and monitoring the complications of feeding. ${ }^{9}$ In addition, patient care might be enhanced by NST through improving nutritional assessment and proper nutrient delivery and reducing infective, mechanical, and metabolic complications. ${ }^{10}$

In this study, we evaluated the impact of NST on postsurgical nutritional status and surgery outcomes in patients with congenital anomalies who underwent repair operation in both NST-supported and not supported NICUs in Dr. Sheikh Pediatric Hospital, Mashhad, Iran.

\section{MATERIALS AND METHODS}

\section{Study design}

This was a retrospective clinical study carried out at two NICUs in Dr. Sheikh Pediatric Hospital, Mashhad, Iran. Each NICU has 12 beds to care for premature neonates and manage associated complications for neonates who require constant close monitoring. Specialist equipment, medications, and trained staff are available 24 hours a day in both NICUs. The patients were operated on by a single surgeon or surgery team. In 2014, NST included physicians, dietitians (two staffs, one Ph.D. student, and two MSc students) and nurses were resided in one NICU cooperating with other NICU staff to supply adequate nutrition support for the patients. Activities of NST included daily, weekly, and monthly programs, which are shown in figure 1.

A total of 120 patients were studied through a purposive 
sampling including 60 patients in NST-supported NICU and 60 patients in the other NICU. Included patients were those aged less than 6 months who had a history of congenital GI anomalies and consequent correction surgery. All medical documents were collected in the archive section of the hospital from initiation of NST in December 2010. In the current study, target GI anomalies included esophageal atresia, imperforated anus, Hirschsprung's disease, malrotation, Meckel's diverticulum, jejunal atresia, duodenal atresia, and diaphragmatic hernia. Patients who experienced more than one surgery during NICU stay or had simultaneous disease or complications and those whose medical files or dietary information had lost were excluded.

The protocol of this study was approved by the hospital's Ethics Committee of Mashhad University of Medical Sciences.

\section{Variables}

Data were collected by two MSc students of nutrition sciences through filling a retrospective chart review and checking variables in three domains of demographic, treatment outcome, and nutrition.

Demographic variables included age, sex, prematurity, birth weight, the highest level of plasma glucose during admission, and type of anomalies. Moreover, the duration of mechanical ventilation that suggests the difficulty in spontaneous breathing and vasoactive drug use (dopamine or dobutamine) were investigated in both NICUs. Vasoactive drugs are used for treating shock and low blood pressure caused by surgery. They are also used to help improve heart function when it is unable to pump enough blood.

Length of stay in NICU, enteral nutrition initiation after surgery, mortality rate, and weight gain in NICU were recorded as outcome variables. Nutrition-related variables consisted of the percentage of calorie delivered to calorie requirement, distribution of energy delivered through enteral or parenteral roots, prescribed intralipid, amino acid, dextrose, medium-chain triglycerides (MCT) oil, as well as breast milk feeding rate during seven days after surgery.

We used the following ratio to calculate the calorie requirement for each patient: ${ }^{11}$

Premature neonates: $120 \mathrm{kcal} / \mathrm{kg} \times 1.2$ (stress factor related to surgery)

Mature neonates: $100 \mathrm{kcal} / \mathrm{kg} \times 1.2$ (stress factor related to surgery)

The average delivered calories per day were calculated by dividing total calorie intake during NICU stay to the number of days. A maximum of seven days of nutrition record was considered for those patients who needed a longer stay.

\section{Statistical analyses}

Analyses were conducted using the Statistical Package for Social Sciences (version 15.0; SPSS Inc, Chicago IL). The normality of data was evaluated using the KolmogorovSmirnov test. The characteristics of subjects at baseline were expressed as mean $\pm \mathrm{SD}$ and median (interquartile 25-75) for continuous variables or percentages for categorical variables. Independent sample t test and Mann-Whitney test were used to compare continuous variables between the two NICUs and Chi-square test was used for categorized variables. The statistical significance level was set at $p<0.05$.

\section{RESULTS}

A total of 120 patients were enrolled in this study (60 patients in each NICU). The demographic characteristics of the patients admitted to both NICUs are shown in table1. There was no statistically significant difference between NST-supported and non-supported NICUs with respect to age, sex, birth weight, weight at the entrance to NICU, prematurity, mean serum glucose, use of vasoactive drugs, and duration of mechanical ventilation. Distribution of congenital GI anomalies in patients of both NICUs showed variation in the pattern of anomalies; although, the frequency of different anomalies was not statistically significant among the patients $(p=0.613)$. Evaluation of illness severity was assessed by using PRISM score and there was no significant difference between the two NICUs in expected mortality rate (data are not shown). Results of comparing variables regarding treatment outcomes in patients of the two groups are presented in table 2. Patients admitted to NST-supported NICU had higher weight gain compared with their counterparts in another NICU (110 vs. 65 grams; $p=$ $0.003)$. There was no significant difference in length of NICU stay ( $p=0.622)$, day of enteral nutrition initiation 
Table 1: General characteristics of the patients*

\begin{tabular}{|c|c|c|c|}
\hline Variables & $\begin{array}{l}\text { Nutritionally supported } \\
\text { NICU }(n=60)\end{array}$ & $\begin{array}{l}\text { Nutritionally non-supported } \\
\text { NICU }(n=60)\end{array}$ & $p$ value $^{2}$ \\
\hline Age (day) & $8.0(4.0-12.0)$ & $7.5(3.0-30.5)$ & 0.692 \\
\hline Sex (Male \%) & 49.2 & 45.2 & 0.579 \\
\hline Birth weight (gr) & $2817 \pm 795$ & $2589 \pm 745$ & 0.108 \\
\hline Weight when admitted to NICU (gr) & $2855 \pm 910$ & $2710 \pm 884$ & 0.380 \\
\hline Prematurity \% & 14.8 & 22 & 0.305 \\
\hline Highest level of serum glucose (mg/dL) & $98.0(66.0-132.0)$ & $95.0(78.0-153.5)$ & 0.438 \\
\hline Vasoactive drugs use $\%$ & 14.8 & 16.9 & 0.550 \\
\hline Duration of ventilation (day) & $2(1-5)$ & $2(1-4)$ & 0.280 \\
\hline Anomalies & & & 0.613 \\
\hline
\end{tabular}

*Mean \pm SD or median (IQ 25-75) for continuous variables or percent for categorical variables.

Table 2: Changes in treatment outcome after implementation of NST*

\begin{tabular}{|c|c|c|c|}
\hline Variables & $\begin{array}{c}\text { Nutritionally supported } \\
\text { NICU }\end{array}$ & $\begin{array}{l}\text { Nutritionally non-sup- } \\
\text { ported NICU }\end{array}$ & $p$ value $^{2}$ \\
\hline Length of NICU stay (day) & $6.0(4.0-9.5)$ & $6.0(4.0-8.0)$ & 0.622 \\
\hline Enteral nutrition initiation after surgery (day) & $4.54 \pm 2.60$ & $4.29 \pm 3.2$ & 0.642 \\
\hline Mortality (\%) & 11.5 & 11.9 & 0.940 \\
\hline Weight gain (gr) & $110(35-255)$ & $65(0-110)$ & 0.003 \\
\hline
\end{tabular}

*Mean \pm SD or median (IQ 25-75) for continuous variables or percent for categorical variables

after surgery $(p=0.642)$, and percent of mortality between the two groups $(p=0.940)$.

Calculating calorie requirements of term and preterm neonates in the two NICUs did not show any significant difference; however, calorie prescribed in NST-supported NICU was significantly higher $(p<0.001)$. The percentage of calories prescribed to calorie required in the educational NICU was significantly more $(p=0.001)$. Among the preterm neonates there was no significant difference in this context (table 3 ).

Calorie intakes from enteral and parenteral support nutrition are shown in table 4. Calorie intake from dextrose in non-supported NICU was significantly greater than NST-supported NICU $(p=0.030)$, while the percentage of energy intakes from intra-lipid ( $p=0.043$ ), and amino acid $(p=0.010)$ in NST-supported NICU was more than the other NICU.

\section{DISCUSSION}

In this study, we observed that NST roles in nutritionally supported NICU improved post-surgery weight gain, and nutritional adequacy and led to a reduction in the use of parenteral nutrition. In addition, parenteral aminoacid and intra-lipid were administrated more frequently than dextrose in NST-supported NICU.

According to the American Society for Enteral and Parenteral Nutrition (ASPEN) report, the most common variable assessed by nutrition support teams is the percent of energy intake compared with the needs. ${ }^{8}$ In a similar study, Mo and colleagues showed that the presence of NST in adult ICU increased nutritional efficiency around $100 \%{ }^{7}$ and a clinical audit that was done in two hospitals for acute care in Ayrshire, one with nutritional support team and the other without support, indicated that establishment of nutrition support team led to a closer nutritional assessment and nutritional efficacy. ${ }^{12}$ In our study, the nutritional adequacy for term neonates in NST-supported NICU was significantly more than other NICU and this difference was due to the complexity of nutritional management in NICU, which definitely needs a professional team for management; although the result was not significant for preterm neonates, which is possibly because of the small number of preterm newborns in each group $(9$ in NST-supported and 11 in NST non-supported NICUs). Overall, the patients admitted to NST-supported NICU had higher weight gain compared with their counterparts 
Table 3: Estimated energy requirement for term and preterm neonates*

\begin{tabular}{lccc}
\hline Variables & $\begin{array}{c}\text { Nutritionally supported } \\
\text { NICU }\end{array}$ & $\begin{array}{c}\text { Nutritionally non-sup- } \\
\text { ported NICU }\end{array}$ & $\boldsymbol{P}^{\text {value }}{ }^{2}$ \\
\hline Term neonates & & & $361.1 \pm 88.8$ \\
\hline Energy requirement (kcal) & $359.5 \pm 92.7$ & $133.9 \pm 45.9$ & 0.930 \\
\hline Energy prescribed (kcal) & $176 \pm 53.4$ & 39 & $<0.001$ \\
\hline Energy prescribed/required (\%) & 50 & & $<0.001$ \\
\hline Preterm neonates & $293.9 \pm 98.5$ & $237.9 \pm 59.7$ & 0.300 \\
\hline Energy requirement (kcal) & $112.25 \pm 44.3$ & $96.68 \pm 43$ & 0.426 \\
\hline Energy prescribed (kcal) & 42 & 39 & 0.652 \\
\hline Energy prescribed/required (\%) & & &
\end{tabular}

Table 4: Distribution of energy intake from enteral and parenteral nutrition*

\begin{tabular}{lccc}
\hline Variables & $\begin{array}{c}\text { Nutritionally supported } \\
\text { NICU }\end{array}$ & $\begin{array}{c}\text { Nutritionally non-sup- } \\
\text { ported NICU }\end{array}$ & $\boldsymbol{P}^{\text {value }}{ }^{2}$ \\
\hline Energy from dextrose (\%) & $58.9 \pm 16.4$ & $65.7 \pm 17.2$ & 0.030 \\
\hline Energy from aminoacid (\%) & $9.6(6.6-13.3)$ & $8.07(4.7-10.5)$ & 0.010 \\
\hline Energy from intralipid (\%) & $17.3(10.7-28.5)$ & $15.3(5.4-15.3)$ & 0.043 \\
\hline Energy from breast-milk (\%) & $6.7(1.9-18.4)$ & $3.9(1.5-16.8)$ & 0.330 \\
\hline${ }^{*}$ Mean \pm SD or median (IQ 25-75) for continuous variables & & &
\end{tabular}

in another NICU because NST had set nutritional support goals based on the infant growth and development to reduce malnutrition and used daily revision and routine examination of energy and macronutrient intake by dietitians.

NST functions in nutritionally supported NICU led to a reduction in the use of parenteral nutrition and increased use of enteral nutrition; therefore, calorie intake from breastfeeding was also increased in NST-supported NICU, although the difference was not significant. Neonates with congenital GI anomalies are at high risk of postoperative nutritional deprivation. This is because of significant challenges to enteral feeding in this group. Some of these surgeries may require a period of gut rest. Hence, nutrient delivery from the enteral root in this vulnerable group needs special attention, which provides by NST in our NICU.

According to guidelines, the team emphasized that enteral nutrition should be preferred over parenteral nutrition even among critically ill children or surgical patient who did not have contraindications for enteral nutrition. ${ }^{13}$ Metabolic and hepatobiliary complications, catheter-related infection, and sepsis are the most prevalent complications of parenteral nutrition. ${ }^{11,13}$ On the other hand, parenteral nutrition increases oxygen free radicals in postoperative infants, which is probably due to intralipid administration. Enteral nutrition is associated with improving clinical outcomes; however, it may be restricted by GI complications and intolerance. Gastric residue should be checked before each feeding. ${ }^{14}$ The presence of NST in other studies was associated with an increased percent of prescribed calorie from enteral nutrition and resulted in the reduction of hospital cost and mortality rate. ${ }^{10,15}$

We checked the regimen of parenteral nutrition in two NICUs and observed that aminoacid and intralipid were more likely to be used in NST-supported NICU while patients in the non-supported NICU were prescribed significantly more energy from dextrose. Studies showed that metabolic and endocrine response after surgeries drive stress hormones and decrease secretion and activities of insulin hormone. ${ }^{16,17}$ This condition motivated hyperglycemia and protein breakdown, which occurs more in children than adults after surgery. Studies on the metabolic response in infants who undergo surgery indicate that protein turnover is influenced by fat to carbohydrate ratio, ${ }^{5}$ and parenteral nutrition support with aminoacid rather than dextrose is a useful method to control this state. ${ }^{18}$

In the current study, NST could not decrease enteral 
nutrition initiation days after surgery, duration of mechanical ventilation, and length of NICU stay. The mortality rate in NST-supported NICU was less than the other NICU, although without statistical significance. Nutritional investigation performed by Ekingen and colleagues in neonates with congenital anomalies after repair surgery showed that early enteral nutrition resulted in a shorter duration of NICU stay and nasogastric tube drainage, decreased time of first stool passage and full oral feeding tolerance. ${ }^{19}$

The current study has some limitations. The first was the small sample size to analyze the outcome variables for each type of GI anomalies. Future research is suggested to study the outcomes of different surgical congenital GI abnormalities after the implementation of NST. Another limitation was that the students of nutrition in NICUs were replaced after the training course.

\section{CONCLUSION}

This survey showed the effectiveness of NST on the clinical outcome of patients with congenital GI anomalies. Neonates undergoing major surgeries need more attention and support, not only for healing but also for optimal growth and development. The presence of NSTs in NICUs can increase weight gain after surgery, as well as improving calorie delivery to the patients. The implementation of nutritional support can also increase enteral nutrition rather than parenteral nutrition for the distribution of calorie intake.

\section{ETHICAL APPROVAL}

There is nothing to be declared.

\section{CONFLICT OF INTEREST}

The authors declare no conflict of interest related to this work.

\section{REFERENCES}

1. Fotis L, Burns AJ, Thapar N. Gastrointestinal Tract: Congenital Abnormalities. eLS 2012. doi: 10.1002/9780470015902.a0002138.pub2

2. Mohammadzadeh A, Farhat A. Gastrointestinal Anomalies in Imam Reza Hospital Deliveries-Mashhad Iran. Int J Pediatrics 2014;2:58. doi: 10.22038/ijp.2014.2519.

3. Burjonrappa SC, Miller M. Role of trace elements in parenteral nutrition support of the surgical neonate. $J$ Pediatr
Surg 2012;47:760-71. doi: 10.1016/j.jpedsurg.2012.01.015.

4. Smith GA. Nutritional Support of the Hospitalized Child. $A d v$ Nutr Res 1983;5:77-103. doi: 10.1007/978-1-4613-9937-7_5

5. Pierro A. Metabolism and nutritional support in the surgical neonate. J Pediatr Surg 2002;37:811-22. doi: 10.1053/jpsu.2002.32879.

6. Kennedy JF, Nightingale JM. Cost savings of an adult hospital nutrition support team. Nutrition 2005;21:112733. doi: 10.1016/j.nut.2005.08.002.

7. Mo YH, Rhee J, Lee E-K. Effects of nutrition support team services on outcomes in ICU patients. Yakugaku Zasshi 2011;131:1827-33. doi: 10.1248/yakushi.131.1827.

8. DeLegge M, Wooley JA, Guenter P, Wright S, Brill J, Andris D, et al. The state of nutrition support teams and update on current models for providing nutrition support therapy to patients. Nutr Clin Pract 2010;25:76-84. doi: 10.1177/0884533609354901.

9. Schneider PJ. Nutrition support teams: an evidencebased practice. Nutr Clin Pract 2006;21:62-7. doi: 10.1177/011542650602100162.

10. Jeong E, Jung YH, Shin SH, Kim MJ, Bae HJ, Cho YS, et al. The successful accomplishment of nutritional and clinical outcomes via the implementation of a multidisciplinary nutrition support team in the neonatal intensive care unit. BMC Pediatr 2016;16:113. doi: 10.1186/ s12887-016-0648-0.

11. Herman R, Btaiche I, Teitelbaum DH. Nutrition support in the pediatric surgical patient. Surg Clin North Am 2011;91:511-41. doi: 10.1016/j.suc.2011.02.008.

12. Fettes SB, Lough M. An audit of the provision of parenteral nutrition in two acute hospitals: team versus non-team. Scott Med J 2000;45:121-5. doi: 10.1177/003693300004500409.

13. Guidelines for the use of parenteral and enteral nutrition in adult and pediatric patients. JPEN J Parenter Enteral Nutr 2002;26(1 Suppl):1SA-138SA.

14. Btaiche IF, Chan LN, Pleva M, Kraft MD. Critical illness, gastrointestinal complications, and medication therapy during enteral feeding in critically ill adult patients. Nutr Clin Pract 2010;25:32-49. doi: $10.1177 / 0884533609357565$.

15. Mehta NM, Skillman HE, Irving SY, Coss-Bu JA, Vermilyea S, Farrington EA, et al. Guidelines for the provision and assessment of nutrition support therapy in the pediatric critically ill patient: Society of Critical Care Medicine and American Society for Parenteral and Enteral Nutrition. JPEN J Parenter Enteral Nutr 2017;41:706-742. doi: 10.1177/0148607117711387.

16. Feldheiser A, Aziz O, Baldini G, Cox B, Fearon K, Feldman L, et al. Enhanced Recovery After Surgery 
(ERAS) for gastrointestinal surgery, part 2: consensus statement for anaesthesia practice. Acta Anaesthesiol Scand 2016;60:289-334. doi: 10.1111/aas.12651.

17. Weimann A, Braga M, Carli F, Higashiguchi T, Hübner $\mathrm{M}$, Klek S, et al. ESPEN guideline: Clinical nutrition in surgery. Clin Nutr 2017;36:623-50. doi: 10.1016/j. clnu.2017.02.013.

18. Donatelli F, Schricker T, Mistraletti G, Asenjo F, Parrella $\mathrm{P}$, Wykes L, et al. Postoperative infusion of amino acids induces a positive protein balance independently of the type of analgesia used. Anesthesiology 2006;105:253-9. doi: 10.1097/00000542-200608000-00007.

19. Ekingen G, Ceran C, Guvenc BH, Tuzlaci A, Kahraman $\mathrm{H}$. Early enteral feeding in newborn surgical patients. Nutrition 2005;21:142-6. doi: 10.1016/j.nut.2004.10.003. 